\title{
Chronic Spontaneous Urticaria - an evaluation of an indirect immunofluorescence method for detecting anti-mast cell IgG antibodies
}

\author{
Bahar Bahrani", Natasha Gattey, Peter Hull \\ From Canadian Society of Allergy and Clinical Immunology Annual Scientific Meeting 2013 \\ Toronto, Canada. 3-6 October 2013
}

\section{Background}

An autoimmune basis is believed to be responsible for about half of all cases of chronic spontaneous urticaria (CSU) with specific IgG antibodies directed at the high affinity receptor sites for IgE on the mast cell. The autologous serum skin test (ASST) is used to identify this autoimmune form of CSU. Currently, basophil histamine release assay and basophil activation test (BAT) have been used as an alternative to the ASST. These tests are not widely available and are limited in that they only provide evidence that the patient's serum is capable of inducing basophil degranulation.

We have developed an indirect immunofluorescence method to demonstrate the presence of anti-mast cell antibodies using skin sections from a patient with severe bullous mastocytosis.

\section{Methods}

Sections were cut from paraffin embedded blocks from skin biopsied infant with bullous mastocytosis. An EDTA buffer solution for heat-induced epitope retrieval was used. Serum from 69 patients with CSU was used, and fluorescein conjugated human IgG was used to label fixed antibody. An ASST had been previously performed on 66 of the patients with severe urticaria and was found to be positive in $45.45 \% .27$ of these patients were receiving intravenous immunoglobulin (IVIG) or had received in the past.

\section{Results}

A positive indirect immunofluorescence was found in half the patients. It was positive in $22.73 \%$ of the patients with a positive ASST, but was also positive in $25.76 \%$ with a negative ASST. The sensitivity and specificity of ASST were calculated to be $46.88 \%$ and $52.94 \%$, respectively. We considered the possibility that the use of IVIG might interfere with indirect immunofluorescence, and this subset was omitted giving a sensitivity and specificity of $34.62 \%$ and $77.27 \%$, respectively.

\section{Conclusion}

Positive indirect immunofluorescence was found in half the patients with CSU. When IVIG treated patient were excluded the ASST was associated with is a high specificity but with low sensitivity. Indirect immunofluorescence should be considered as better indicator of the autoimmune form of urticaria.

Published: 3 March 2014

doi:10.1186/1710-1492-10-S1-A2

Cite this article as: Bahrani et al:: Chronic Spontaneous Urticaria - an evaluation of an indirect immunofluorescence method for detecting anti-mast cell IgG antibodies. Allergy, Asthma \& Clinical Immunology 2014 10(Suppl 1):A2.

\footnotetext{
* Correspondence: Bab210@mail.usask.ca

Department of Medicine, University of Saskatchewan, Saskatoon,

Saskatchewan, Canada, S7N OW8
} 